\title{
Editorial: Phenomena Beyond the Standard Model: What Do We Expect for New Physics to Look Like?
}

\author{
António P. Morais ${ }^{1}$, Stefano Moretti ${ }^{2 \star}$ and Roman Pasechnik ${ }^{3}$ \\ ${ }^{1}$ Departamento de Física, Universidade de Aveiro and CIDMA - Center for Research and Development in Mathematics and \\ Applications, Aveiro, Portugal, ${ }^{2}$ School of Physics and Astronomy, University of Southampton, Southampton, \\ United Kingdom, ${ }^{3}$ Department of Astronomy and Theoretical Physics, Lund University, Lund, Sweden
}

Keywords: Higgs physics, grand unified theories, dark matter, baryogenesis, cosmic inflation

\section{Editorial on the Research Topic}

Phenomena Beyond the Standard Model: What Do We Expect for New Physics to Look Like?

OPEN ACCESS

Edited and reviewed by: J.W. F. Valle,

Consejo Superior de Investigaciones Cientificas (CSIC), Spain

*Correspondence:

Stefano Morett

s.moretti@soton.ac.uk

Specialty section:

This article was submitted to High-Energy and Astroparticle

Physics,

a section of the journal

Frontiers in Physics

Received: 10 May 2020

Accepted: 18 May 2020

Published: 21 July 2020

Citation:

Morais AP, Moretti S and Pasechnik R (2020) Editorial: Phenomena Beyond

the Standard Model: What Do We

Expect for New Physics to Look Like?

Front. Phys. 8:209

doi: 10.3389/fphy.2020.00209
Particle physics (PP) is a vast and active research field of contemporary theoretical and experimental physics. Measurements made at microscopic distances have started to confront the most fundamental principles of nature encoded in the structure of the Standard Model (SM) of PP. With recent observations of accelerated expansion of the universe, massive dark halos filled with invisible matter, and persistent flavour physics anomalies, the SM is entering a period of most severe phenomenological tests that could eventually lead to a revision of our current understanding of the fundamental properties of matter, interactions, and even spacetime.

While Large Hadron Collider (LHC) experiments have accessed fundamental interactions at the energy and intensity frontiers without notable discoveries so far, the demand for precision measurements is increasing. Already we are familiar with persistent inconsistencies within the SM framework, such as the absence of a viable dark matter (DM) candidate, the failure to describe the origin of dark energy, the inability to account for sufficient CP violation required for generation of the baryon asymmetry, the yet-to-be resolved hierarchy problem in the Higgs sector, and the lack of a dynamical mechanism for the natural generation of very specific observed patterns in fermion mass and mixing parameters. For instance, there is a substantial lack of first-principles understanding of the Higgs sector properties and origin of the electroweak scale, of the three quark/lepton families, of the unique neutrino features, and of the strong unexplained hierarchies in the lepton and quark sectors of the SM. The non-observation of New Physics (NP) in collider measurements remains puzzling and raises questions as to their discovery potential, methodology, precision, and sensitivity to weak signals. Conversely, with a wealth of new phenomenological information emerging from neutrino oscillation studies, astroparticle physics measurements, lowenergy analyses, and, more recently, gravitational waves, can we expect the SM to remain the baseline framework of PP, or should it be replaced eventually by a more accurate and complete theory of the building blocks and symmetries of nature? What kind of NP can we expect to show up and in what particular way?

This Research Topic "Phenomena Beyond the Standard Model: What Do We Expect for New Physics to Look Like?" is devoted to highlighting selected topics in state-of-the-art theoretical 
research at the forefront of $\mathrm{PP}$ that explores these most fundamental questions of nature, in the quest for some Beyond the Standard Model (BSM) phenomena. A total of ten papers were submitted, covering exciting developments in the direction of uncovering some NP from both the theoretical and the experimental sides. On the one hand, two paradigms for solving the hierarchy problem of the SM, Supersymmetry and Compositeness, are considered. On the other hand, experiments from low to high energy scales are discussed, in settings ranging from colliders to ground as well as space apparatus. Delle Rose et al. address the case of potential NP signals at energies as low as $17 \mathrm{MeV}$, while Croon et al. discuss the properties of a prototypical Grand Unified Theory (GUT) at $10^{16} \mathrm{GeV}$. As two major flaws of the $\mathrm{SM}$ are that, therein, neutrinos are massless and there is no viable candidate for DM, it is no surprise that Shindou considers the first case while a number of other authors (Bhattacharya et al.; Belyaev; Corianò et al.; Khlopov) address the second one, including discussing the key phenomenological consequences of the corresponding BSM constructs in the aforementioned experimental settings. Finally, given the importance of the Higgs boson discovery at CERN in 2012 for the whole of PP, it is natural to also see several submissions addressing the possibility of a BSM origin of this crucial particle, within extended Higgs sectors whose motivation (as explained by Miller et al.) builds upon the Multiple Point Principle (MPP), indicating their plausibility, including both the cases of Supersymmetry (Arhrib et al.) and Compositeness (Cacciapaglia et al.). Altogether, this special issue of Frontiers serves to inform the reader that even though NP is currently unknown to us, we are wellequipped on both the theoretical and the experimental fronts to extract its possible manifestations, whatever these might look like.

\section{AUTHOR CONTRIBUTIONS}

All authors listed have made a substantial, direct and intellectual contribution to the work, and approved it for publication.

\section{FUNDING}

AM was supported by the COST Action CA16201, by the Center for Research and Development in Mathematics and Applications (CIDMA) through the Portuguese Foundation for Science and Technology (FCT -Fundação para a Ciência e a Tecnologia), references UIDB/04106/2020 and UIDP/04106/2020 and by national funds (OE), through FCT, I.P., in the scope of the framework contract foreseen in the numbers 4,5 , and 6 of the article 23, of the Decree-Law 57/2016, of August 29, changed by Law 57/2017, of July 19. AM is also supported by the projects PTDC/FIS-PAR/31000/2017 and CERN/FISPAR/0027/2019. SM was financed through the NExT Institute and the STFC Consolidated Grant No. ST/L000296/1. RP was partially supported by the Swedish Research Council, grant numbers 621-2013-4287 and 2016-05996, as well as the ERC grant agreement No. 668679.

Conflict of Interest: The authors declare that the research was conducted in the absence of any commercial or financial relationships that could be construed as a potential conflict of interest.

Copyright (๑) 2020 Morais, Moretti and Pasechnik. This is an open-access article distributed under the terms of the Creative Commons Attribution License (CC BY). The use, distribution or reproduction in other forums is permitted, provided the original author(s) and the copyright owner(s) are credited and that the original publication in this journal is cited, in accordance with accepted academic practice. No use, distribution or reproduction is permitted which does not comply with these terms. 